\title{
Commentary: Not cool enough-Surgical cryoablation during ventricular reconstruction
}

\author{
Ammar Killu, MBBS, ${ }^{a}$ and Juan A. Crestanello, MD $^{\mathrm{b}}$
}

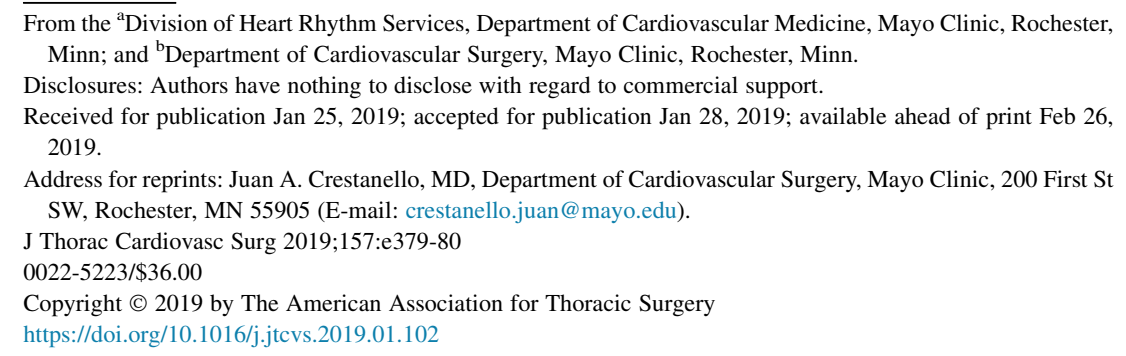

Cardiac surgeons have long ablated cardiac arrhythmias. Early experience was gained through accessory pathway ablation and moved toward atrial and ventricular arrhythmias. Multiple energy sources have been used, including physical interruption or division, radiofrequency, and, more recently, cryoablation, all aiming to create zones of electrical nonconductivity and thereby preventing propagation of electrical impulses across the lesion line. Although catheter ablation has circumvented the need for surgical ablation in many cases, certain situations provide a unique opportunity in which surgical ablation may improve patient outcomes. ${ }^{1}$

After infarction, progressive cardiac remodeling may result in left ventricular (LV) aneurysm formation. Because of the deleterious effect on cardiac function, Dor and colleagues ${ }^{2}$ devised the eponymous procedure to exclude the noncontracting aneurysmal segment and reduce ventricular size. Through the years, this procedure has undergone various modifications. Despite these refinements, patient outcome remains suboptimal because of malignant ventricular arrhythmias, which have been mapped both to the LV aneurysm wall (to the transition zone between scar regions and viable myocardium) and also to remote myocardium. Although the former can be explained by areas of fibrosis interspersed with viable myocardium, resulting in unidirectional block and sufficiently slow conduction, thereby setting the substrate for reentry, the latter is more difficult to explain. Regions of scar may, however, exist outside the aneurysm. In addition, eccentric hypertrophy and dilation of nonaneurysmal regions increase wall stress and have a deleterious effect on oxygen supply and demand relationship, facilitating arrhythmogenesis. ${ }^{3}$

Given the high incidence of malignant arrhythmias, addition of ablation of the LV border zone and endocardectomy during surgical ventricular reconstruction has been advocated. ${ }^{4,5}$ The results of these procedures have been suboptimal, however, and the rate of arrhythmia recurrence remains high, with most patients benefiting from been difficult.

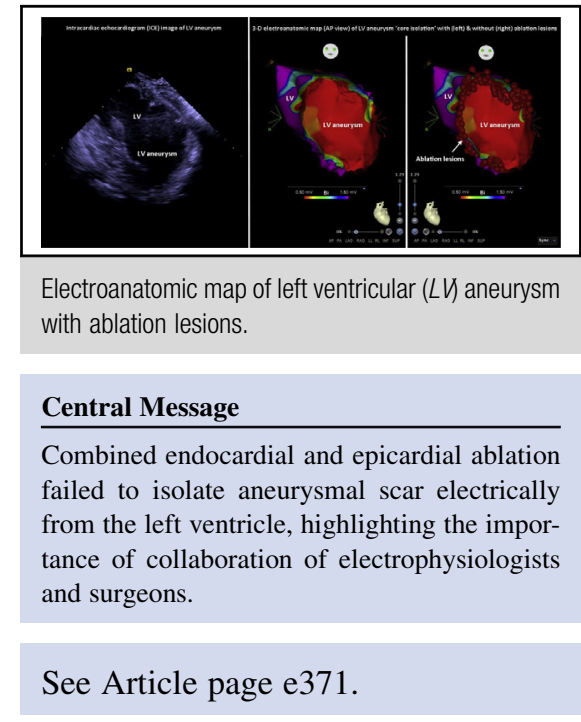

implantable cardioverter defibrillator placement. Although electrical isolation of the border zone from the remaining "healthy" ventricular myocardium is an appealing concept to prevent propagation of the arrhythmias, execution has

In the current issue of the Journal, van Huls van Taxis and colleagues ${ }^{6}$ recognize the deficient outcomes associated with endocardial cryoablation at the time of surgical ventricular reconstruction. They conducted a randomized study assessing the effectiveness of endocardial cryoablation alone versus combined endocardial and epicardial cryoablation in isolating the aneurysmal scar.

Unfortunately, results of the trial were negative, and it was aborted because neither endocardial cryoablation alone nor combined endocardial and epicardial ablation was able to isolate the scar tissue electrically from the ventricular wall. Furthermore, ventricular tachycardia (VT) recurrence was high in both groups-which was unsurprising, given that scar isolation was not achieved! Certainly, partial ablation can result in conduction slowing that may actually promote arrhythmogenesis.

Although results of the trial were negative, several questions arise:

1. Is the electrical isolation of the LV aneurysm scar from the remaining myocardium effective? Theoretically, if the aneurysm is critical to generation of VT, then complete exclusion should abolish the arrhythmia. Indeed, 
core isolation in which an area is electrically isolated from its surround myocardium has been used to good effect in catheter ablation cases with multiple or unmappable VT, especially if exit block (in essence, electrical isolation) is achieved. ${ }^{7}$ Although endocardial catheter ablation alone is unlikely to create transmural lesions, it may sufficiently alter the electrophysiologic milieu and therefore reduce the risk for arrhythmogenesis.

2. Is there another, more effective technique? In catheter ablation, cryoablation has been shown to carry a higher recurrence rate than radiofrequency ablation. Cryoablation can achieve effective ablation through several cellular effects, however, including ion channel dysfunction and ice crystal formation resulting in desiccation. The fact that no conduction delay was noted suggests that ablation was inadequate. As noted previously, transmural lesions are difficult to achieve. Whether an alternative approach, such as combining subendocardial resection in addition to cryoablation, using freezethaw-freeze cycles, or extending the time of freezing until electrical isolation is achieved, would have yielded higher success is undetermined. ${ }^{8}$ Although radiofrequency ablation might have been more effective, electroporation (nanosecond or microsecond electrical pulses) may hold promise in the future.

3. Is there a role for a hybrid approach? Patients enrolled in this study all had inducible VT before the surgery, but no electroanatomic mapping-guided ablation was attempted. Empirical surgical ablation is note guided, and although the rapid VT rate typically seen in these patients precludes activation and entrainment mapping, substrate mapping before surgery may help delineate abnormal regions of myocardium in nonaneurysmal regions. ${ }^{9}$ Further, presurgical catheter ablation before surgical cryoablation may facilitate achievement of core isolation. Importantly, exclusion of the aneurysm with patch placement can bar percutaneous access to the critical regions of myocardium should catheter ablation be attempted at a later date.

4. Would image-guided empirical ablation be beneficial? Scar may be difficult to accurately discern intraoperatively. Empirical ablation of scar regions seen on cardiac imaging (eg, magnetic resonance imaging), may be beneficial. $^{10}$

5. Should histologic analysis be performed? Because of the study methods, no histologic analysis was possible, although cardiac autopsy from deceased patients might have provided a unique insight into the extent and effect of the cryoablation methods used.

Given the high rate of ventricular arrhythmias, cardioverter defibrillator implantation should at least be considered, if not mandated, before hospital discharge. Although results of the study were negative, the work of van Huls van Taxis and colleagues ${ }^{6}$ serves as a reminder of the importance of collaboration, namely between invasive electrophysiologists and cardiac surgeons, to improve arrhythmia outcomes in patients undergoing complex cardiac surgery.

\section{References}

1. Wellens F, Geelen P, Demirsoy E, Van Praet F, De Geest R, Degrieck I, et al. Surgical treatment of tachyarrhythmias due to postinfarction left ventricular aneurysm with endoaneurysmorrhaphy and cryoablation. Eur J Cardiothorac Surg. 2002;22:771-6.

2. Dor V, Saab M, Coste P, Kornaszewska M, Montiglio F. Left ventricular aneurysm: a new surgical approach. Thorac Cardiovasc Surg. 1989;37:11-9.

3. Athanasuleas CL, Stanley AW Jr, Buckberg GD, Dor V, DiDonato M, Blackstone EH. Surgical anterior ventricular endocardial restoration (SAVER) in the dilated remodeled ventricle after anterior myocardial infarction. RESTORE group. Reconstructive endoventricular surgery, returning torsion original radius elliptical shape to the LV. J Am Coll Cardiol. 2001;37:1199-209.

4. Demaria RG, Mukaddirov M, Rouvière P, Barbotte E, Celton B, Albat B, et al. Long-term outcomes after cryoablation for ventricular tachycardia during surgical treatment of anterior ventricular aneurysms. Pacing Clin Electrophysiol. 2005;28(Suppl 1):S168-71.

5. Babokin V, Shipulin V, Batalov R, Popov S. Surgical ventricular reconstruction with endocardectomy along radiofrequency ablation-induced markings. J Thorac Cardiovasc Surg. 2013;146:1133-8.

6. van Huls van Taxis C, Zeppenfeld K, Klautz R, Wijnmaalen AP. Endocardial/ epicardial versus endocardial encircling cryoablation for ventricular tachycardia: a randomized trial. J Thorac Cardiovasc Surg. 2019;157:e371-7.

7. Tzou WS, Frankel DS, Hegeman T, Supple GE, Garcia FC, Santangeli P, et al. Core isolation of critical arrhythmia elements for treatment of multiple scar-based ventricular tachycardias. Circ Arrhythm Electrophysiol. 2015;8:353-61.

8. Gage AA, Baust J. Mechanisms of tissue injury in cryosurgery. Cryobiology. 1998;37:171-86.

9. Josephson ME, Anter E. Substrate mapping for ventricular tachycardia: assumptions and misconceptions. JACC Clin Electrophysiol. 2015;1:341-52.

10. Piers SR, Zeppenfeld K. Imaging-guided ventricular tachycardia ablation. Arrhythm Electrophysiol Rev. 2013;2:128-34. 